October 26, 2018

\title{
Coherence and oscillations of cosmic neutrinos
}

\author{
Yasaman Farzan $^{a}{ }^{1}$ and Alexei Yu. Smirnov ${ }^{b, c} 2$ \\ ${ }^{a}$ Institute for research in fundamental sciences (IPM), P.O. Box \\ 19395-5531, Tehran, Iran \\ ${ }^{b}$ International Centre for Theoretical Physics, Strada Costiera 11, 34014 \\ Trieste, Italy, \\ ${ }^{c}$ Institute for Nuclear Research, Russian Academy of Sciences, Moscow, \\ Russia
}

\begin{abstract}
For cosmic neutrinos we study the conditions and the effects of the coherence loss as well as coherent broadening of the spectrum. We evaluate the width of the neutrino wavepacket produced by charged particles under various circumstances: in an interaction-free environment, in a radiation-dominated medium (typical of the sources of the gamma ray bursts) and in the presence of a magnetic field. The effect of the magnetic field on the wavepacket size appears to be more important than the scattering. If the magnetic field at the source is larger than $\sim 10$ Gauss, the coherence of neutrinos will be lost while traveling over cosmological distances. Various applications of these results have been considered. We find that for large magnetic fields $\left(B>10^{9}\right.$ Gauss) and high energies $\left(E_{\nu}>\mathrm{PeV}\right)$, "coherent broadening" can modify the energy spectrum of neutrinos. In the coherent case, averaging out the oscillatory terms of the probabilities does not induce any statistical uncertainty beyond what expected in the absence of these terms. A deviation from the standard quantum mechanics that preserves average energy and unitarity cannot alter the picture.
\end{abstract}

PACS: $14.60 . \mathrm{Pq}$

KEYWORDS: Cosmic neutrinos, Coherence, Spectrum

\footnotetext{
${ }^{1}$ yasaman@theory.ipm.ac.ir

${ }^{2}$ smirnov@ictp.it
} 


\section{Introduction}

Construction and operation of the neutrino telescopes open new windows towards exploring the Universe. By studying the neutrinos arriving at these telescopes from sources located at cosmological distances, we can derive unprecedented information on the sources of such neutrinos as well as on the propagation and properties of the neutrinos, themselves.

Operating detectors have not so far detected cosmic neutrinos. The data accumulated during 2000-2004 by the AMANDA experiment shows no indication of neutrino flux from beyond the atmosphere of the Earth [1] which leads to an upper bound on the point source flux

$$
E_{\nu}^{2} \frac{d \Phi_{\nu}}{d E_{\nu}} \lesssim 10^{-8} \mathrm{GeV} \mathrm{cm}^{-2} \mathrm{sec}^{-1} \mathrm{sr}^{-1}
$$

in the energy range between $1.6 \mathrm{TeV}$ and $2.5 \mathrm{PeV}$. This bound implies that the total number of events per year at a $1 \mathrm{~km}^{3}$ scale detector such as ICECUBE [2] cannot be on average larger than $10^{4}$. A host of theoretical models predict cosmic neutrino fluxes in the $(1-100) \mathrm{TeV}$ energy range which can be detected by a $1 \mathrm{~km}^{3}$ scale detector: e.g., the fireball models for Gamma Ray Burst (GRB) sources [3, 4], models for supernovae type I b/c [5, 6] and models for the Active Galactic Nuclei (AGN) [7]. The neutrino flux from GRB in the (1-10) TeV range can saturate the present bound from AMANDA [4]. We should prepare for such generosity of nature and anticipate what can be learned about the neutrino properties as well as the mechanism behind the production of neutrinos at the source. Because of this prospect, we mainly concentrate on neutrinos with energies in the $(1-100)$ TeV range throughout the present paper.

According to the models, neutrinos are produced through the following chain of reactions [8]

$$
\begin{aligned}
& p+\gamma \rightarrow \Delta \rightarrow \pi^{+}+X, \\
& \pi^{+} \rightarrow \nu_{\mu}+\mu^{+} \\
& \mu^{+} \rightarrow \nu_{e}+\bar{\nu}_{\mu}+e^{+} .
\end{aligned}
$$

Along with the process $(2)$, both $\pi^{+}$and $\pi^{-}$can be produced via the $p p$ collisions or via the collision of the protons with the nuclei present in the medium. 
If the interaction rates of $\pi$ and $\mu$ are negligible, the flavor composition of the neutrino flux at the source will be as the following

$$
\left(F_{e}^{0}+F_{\bar{e}}^{0}\right):\left(F_{\mu}^{0}+F_{\bar{\mu}}^{0}\right):\left(F_{\tau}^{0}+F_{\bar{\tau}}^{0}\right)=1: 2: 0,
$$

where $F_{\alpha}^{0}$ and $F_{\bar{\alpha}}^{0}$ respectively denote the fluxes of $\nu_{\alpha}$ and $\bar{\nu}_{\alpha}$.

Neutrino oscillations alter the flavor composition of the neutrino flux during propagation from the source to the detector. If the flavor composition at the source is known, by studying the flavor composition at the detector, one can extract information on the oscillation parameters [9]. In particular in $[10,11]$, as the key part of the program of reconstructing the unitarity triangle and deriving the lepton sector Jarlskog invariant, it was suggested to study the cosmic neutrinos to extract the $U_{\mu 1}$ element of the PontecorvoMaki-Nakagawa-Sakata matrix. There is also rich literature on the possibility of directly determining the Dirac CP-violating phase from the neutrino telescope data [12].

One of the important effects of neutrino propagation which can in principle influence observations is the loss of coherence. The different neutrino mass eigenstates having the same energy have different velocities. Thus, the wavepackets of the mass eigenstates composing a neutrino state will come apart as they propagate. If the traveled distance is so large that these components completely separate from each other, they will cease to interfere at the detector. In the case of coherence loss, the oscillatory terms of the oscillation probabilities disappear. Conversely, in the coherent case, the different mass eigenstates keep interfering and as a result, the oscillatory terms are still present. Of course, even in the latter case, because of the finite energy resolution of the detector and the fact that the sources of different events are located at various distances, the effects of the oscillatory terms average to zero. As discussed in $[13,14]$, the two cases are in practice indistinguishable. In this paper, we discuss in detail under which conditions the coherence is lost. We also evaluate the statistical uncertainty in the presence of the oscillatory terms for the coherent case.

Coherent broadening of the wavepacket can lead to deformation of the energy spectrum which has direct observational consequences. In this paper, we discuss under what circumstances, the effect will be noticeable.

The paper is organized as follows. In sec. 2, we elaborate on the conditions of the coherence loss. In sec. 3, we explore how the interactions of the 
parent particles in the production region can affect the sizes of the emitted wavepackets, and consequently, the loss of coherence. In secs. 4.2 and 4.3, we discuss the applications of our finding for neutrinos from various cosmic and terrestrial sources. Sec. 4.4 is devoted to the discussion of coherent deformation of the energy spectrum. In sec. 4.5, we consider the effects of incomplete averaging. In sec. 4.6, we comment on how a deviation from the standard quantum mechanical evolution of states can affect the results. A summary and the discussion of results are given in sec. 5 .

\section{Loss and restoration of coherence}

\subsection{Coherence loss}

The oscillatory terms from the oscillation probabilities can disappear in two physically distinct situations: (1) loss of coherence; (2) averaging. The first case is related to features of production of neutrinos, whereas the second one - of detection. For the scope of further discussion we first formulate the condition for loss of coherence in the momentum space and then derive the same condition considering the problem in the configuration space. The two approaches are equivalent and give the same result.

The phase of oscillation, $\phi$, equals

$$
\phi(E, L) \equiv \frac{\Delta m^{2} L}{2 E},
$$

where $\Delta m^{2}$ is the mass squared difference and $L$ is the distance between the source and the detector which hereafter will be referred to as the baseline. Let us define $\Delta E_{L}$ as energy difference for which $\phi\left(E-\Delta E_{L}, L\right)-\phi(E, L)=2 \pi$. It is straightforward to show that

$$
\Delta E_{L} \simeq \frac{4 \pi E^{2}}{\Delta m^{2} L}=E \frac{l_{\nu}}{L}
$$

where $l_{\nu}=4 \pi E / \Delta m^{2}$ is the vacuum oscillation length. Neutrinos traveling over cosmological distances are essentially on their mass shells. We can therefore use the dispersion relation: $E^{2}=p^{2}+m^{2}$. Introducing the uncertainty in the energy, $\sigma_{E}$ and in the momentum ${ }^{3}, \sigma_{p}$, we find from this relation

\footnotetext{
${ }^{3}$ From now on, by $\sigma_{p}$ we implicitly mean the width of the wavepacket in the longitudinal direction.
} 
that $E \sigma_{E}=p \sigma_{p}$. Width of the wavepacket in the energy-momentum space is determined by $\sigma_{E}$ and $\sigma_{p}$. Furthermore, since neutrinos are ultrarelativistic $(E \simeq p)$, the uncertainty in energy and momentum are practically equal: $\sigma_{E} \simeq \sigma_{p}$. In what follows we will not distinguish between $\sigma_{E}$ and $\sigma_{p}$. ${ }^{4}$

The interference between the effects of different mass eigenstates disappears if

$$
\sigma_{p}>\Delta E_{L}=\frac{4 \pi E^{2}}{\Delta m^{2} L}=E \frac{l_{\nu}}{L} .
$$

(For an explicit proof of the disappearance of the oscillatory terms see the appendix.) Omitting the numerical factor, $4 \pi$, we can rewrite the condition in Eq. (7) as

$$
\frac{\Delta m^{2} L}{E} \frac{\sigma_{p}}{E} \gg 1
$$

Let us now reconsider the same problem in the configuration space. The size of the wavepacket in the configuration space at the production point is given by the inverse of the uncertainty $\sigma_{p}$ :

$$
\sigma_{x} \sim \frac{1}{\sigma_{p}}
$$

A neutrino of a definite flavor can be considered as a superposition of the wavepackets of different mass eigenstates. Due to the difference between the group velocities of the mass eigenstates,

$$
\Delta v=\frac{\Delta m^{2}}{2 E^{2}},
$$

the corresponding wavepackets separate in the course of propagation. The separation, $d_{L}$, after propagating a distance of $L$ is equal to

$$
d_{L}=\frac{L}{v} \Delta v=L \frac{\Delta m^{2}}{2 E^{2}} .
$$

The separation in the configuration space is complete when

$$
\sigma_{x} \ll d_{L}=L \frac{\Delta m^{2}}{2 E^{2}} .
$$

\footnotetext{
${ }^{4}$ Under certain circumstances, like emission of neutrinos from crystals with Mossbauer effect, the difference between the energy and momentum uncertainties becomes important.
} 
At this point, the overlap of packets and therefore interference effects disappear - the coherence is lost. The condition in (12) is equivalent to the condition in (8) in the energy-momentum space. The inequality (8) as well as (12) is called the condition for "loss of coherence". The coherence length is defined as

$$
L_{c o h}=4 \pi \sigma_{x} \frac{E^{2}}{\Delta m^{2}} .
$$

For $L>L_{c o h}$, the coherence is lost and if the coherence is not restored at the detection (see subsec. 2.2 as well as the appendix), the oscillatory terms disappear from the oscillation probabilities.

For the typical source to detector distance $L=100 \mathrm{Mpc}$, the neutrino energy $E=10 \mathrm{TeV}$ and $\Delta m^{2}=\Delta m_{\text {atm }}^{2}=2.5 \cdot 10^{-3} \mathrm{eV}^{2}$, from Eq. (12) we find that the coherence is lost if the size of the wavepacket satisfies

$$
\sigma_{x} \ll d_{L}=3 \cdot 10^{-3} \mathrm{~cm}\left(\frac{L}{100 \mathrm{Mpc}}\right)\left(\frac{\Delta m^{2}}{2.5 \cdot 10^{-3} \mathrm{eV}^{2}}\right)\left(\frac{10 \mathrm{TeV}}{E}\right)^{2} .
$$

Replacing $\Delta m^{2}$ with $\Delta m_{\text {sol }}^{2}$, we obtain a value for $d_{L}$ that is about 30 times smaller. That is, if the size of the wavepacket is much smaller than $\sim 10^{-4}$ $\mathrm{cm}$, the oscillatory terms given by $\Delta m_{\text {sol }}^{2}$ disappear.

In our consideration we have ignored spread (widening) of each wavepacket in the configuration space due to the presence of different energies/momenta in the packet. For a given mass eigenstate, the group velocities of the components of the wavepacket with $\Delta E=\sigma_{E}$ are different: $\Delta v=\left(m^{2} / E^{2}\right)\left(\sigma_{E} / E\right)$. The spread of the wavepacket over a distance $L$ is therefore equal to

$$
\sigma_{L}=|\Delta v L|=\frac{m^{2}}{E^{2}} \frac{\sigma_{E}}{E} L=\left(\frac{m}{E}\right)^{3} \frac{1}{\sigma_{x} m} L .
$$

Comparing this expression with the separation (11) we find that the condition $d_{L} \gg \sigma_{L}$ implies

$$
\frac{\sigma_{E}}{E} \ll \frac{\Delta m^{2}}{m^{2}}
$$

where $m$ is the heaviest neutrino mass. The minimal value on the RHS corresponds to the degenerate neutrinos. Eq. (16) is fulfilled for all situations under consideration in this paper. That is, the separation of the wavepackets is more important than their spread. 


\subsection{Restoration of coherence}

Separation of the wavepackets does not mean yet that the effects of oscillatory terms are absent in observation. If the time scale of the detection process is larger than the time interval between the arrival of the successive wavepackets composing a single state, the wavepackets still interfere at the detector despite the spatial separation [13]. In other words, if the detector has large enough "time memory" the detection restores the coherence. The coherence is determined both by the production and detection. In this sense, there is a symmetry between the source and detector.

Consider a neutrino wavepacket whose components are completely separated: i.e., $\sigma_{x} \sim \sigma_{p}^{-1} \ll d_{L}$, where $d_{L}$ is given in Eq. (11). If the energy of the neutrino is measured with precision better than $d_{L}^{-1}$, the coherence will be restored [13]. According to the energy-time uncertainty principle, such a measurement takes a time longer than $d_{L} / c$ during which the second component arrives at the detection point. However, in the realistic situations under consideration, restoration of the coherence at detection, which requires determination of energy with precision better than $d_{L}^{-1} \sim 10^{-2} \mathrm{eV}(100 \mathrm{Mpc} / L)\left(2.5 \times 10^{-3} \mathrm{eV}^{2} / \Delta m^{2}\right)(E / 10 \mathrm{TeV})^{2}$ (see Eq. (11)), does not take place. Suppose that a hypothetic detector reaches such a sensitivity; then, for each individual neutrino state, coherence is restored. However, even in this, once we accumulate data from different sources located at various distances, the oscillation pattern will disappear.

\section{Width of the neutrino wavepacket}

In this section, we evaluate the width of a neutrino wavepacket produced through the processes shown in Eq. (3) in an environment such as the relativistic jets which, according to the fireball models, are present in the GRB sources. We discuss the dependence of the wavepacket size on the density and the strength of the magnetic field at the production region. We also discuss if a neutrino produced in such an environment loses its coherence traveling over cosmological distances. 


\subsection{Neutrino produced by decay of "free" pions and muons}

In this subsection, we consider the case in which the parent charged particles (pions and muons) do not undergo any interaction with matter or with the magnetic field before decay. The consideration applies for any environment in which the mean free path and the Larmor radius of the parent particle are much larger than the distance that the charged particle travels before decay. The cosmogenic neutrinos (i.e., the neutrinos that are produced through the interaction of the cosmic rays with the galactic and intergalactic medium) can be considered as an example for realization of such a situation.

The width of the wavepacket of the neutrino in the rest frame of the parent particle can be estimated as

$$
\sigma_{E}^{0} \approx \sigma_{p}^{0} \sim \frac{1}{\tau_{0}}
$$

where $\tau_{0}$ is the lifetime of the parent particle in its rest frame (i.e., for the muon $\tau_{0}=2.2 \times 10^{-6}$ sec while for the pion $\left.\tau_{0}=2.6 \times 10^{-8} \mathrm{sec}\right)$.

Suppose the pion propagates along the $z$-direction; i.e., in the observer frame, the energy-momentum of the pion can be written as $\left(E_{\pi}, 0,0, p_{\pi}\right)$. Let us take the four-momentum of the neutrino in the rest frame of the pion to be

$$
\left(E_{\nu}^{0}, 0, E_{\nu}^{0} \sin \xi, E_{\nu}^{0} \cos \xi\right)
$$

where

$$
E_{\nu}^{0}=\frac{m_{\pi}^{2}-m_{\mu}^{2}}{2 m_{\pi}} \sim m_{\pi} / 4
$$

and $\xi$ is the angle between neutrino momentum in the frame of pion and the momentum of pion in the observer frame. The energy of the neutrino in the observer frame can be then written as $E_{\nu}=\left(E_{\pi} / m_{\pi}\right) E_{\nu}^{0}\left(1+v_{\pi} \cos \xi\right)$ where $v_{\pi}=p_{\pi} / E_{\pi} \simeq 1$ is the velocity of the pion. Performing a Lorentz transformation we find that in this frame

$$
\sigma_{E}=\frac{E_{\pi}}{m_{\pi}}\left(\sigma_{E}^{0}+v_{\pi} \sigma_{p}^{0} \cos \xi\right)=\frac{E_{\nu}}{E_{\nu}^{0}} \sigma_{E}^{0} \sim \frac{4 E_{\nu}}{m_{\pi}} \frac{1}{\tau_{0}} .
$$

Notice that $\sigma_{E} / E_{\nu}$ is Lorentz invariant.

It is instructive to derive these results through alternative approaches. The length of the wavepacket in the rest frame of the pion is equal to $c \tau_{0}$. It 
is straightforward to show that in the observer frame the wavepacket length is Lorentz contracted to

$$
\sigma_{x} \simeq \frac{\tau_{0}}{\gamma(1+\cos \xi)} \simeq \frac{E_{\nu}^{0}}{E_{\nu}} \tau_{0} \sim \frac{m_{\pi}}{4 E_{\nu}} \tau_{0} .
$$

The last equation is for neutrinos emitted in the forward direction $\xi=0$. As expected, Eqs. $(19,20)$ yield $\sigma_{x}=\sigma_{E}^{-1}$.

We can rederive Eq. (20) by using the fact that the number of wave periods in the wavepacket is a Lorentz invariant:

$$
\frac{\sigma_{x}}{\lambda}=\sigma_{x} E_{\nu}=\text { invariant }
$$

In the rest frame of the pion this number is $\tau^{0} p_{\nu}^{0}=\tau^{0} E_{\nu}^{0}$, so

$$
\sigma_{x}=\tau^{0} \frac{E_{\nu}^{0}}{E_{\nu}} \sim \tau^{0} \frac{m_{\pi}}{4 E_{\nu}} .
$$

Neutrinos emitted in the forward direction have higher frequency (energy) but shorter packet (pulse).

Another Lorentz invariant that one can use to determine $\sigma_{x}$ in the observer frame is the number of the oscillation periods before the system loses its coherence. This number is given by the ratio of the coherence length $L_{c o h}$ to the oscillation length, $l_{\nu}$. Using Eq. (13), we find

$$
\frac{L_{c o h}}{l_{\nu}}=\sigma_{x} E_{\nu}=\text { invariant }
$$

which immediately leads to the same result as (21).

Numerically, for neutrinos produced by pions we obtain

$$
\sigma_{x} \sim 2 \times 10^{-3} \mathrm{~cm}\left(\frac{10 \mathrm{TeV}}{E_{\nu}}\right) .
$$

For the neutrinos produced by muons, $\sigma_{x}$ is larger by the ratio of the lifetime of the muon to that of the pion: $\sigma_{x} \sim 0.2 \mathrm{~cm}\left(10 \mathrm{TeV} / E_{\nu}\right)$.

Let us consider the ratio of separation length and the length of the wavepacket which, as discussed in sec. 2.1, determines the loss of coherence:

$$
\frac{d_{L}}{\sigma_{x}}=\frac{\Delta m^{2} L}{2 E_{\nu}^{2}} \frac{1}{\sigma_{x}} \sim \frac{\Delta m^{2} L}{E_{\nu}} \frac{2}{m_{\pi} \tau^{0}} .
$$


(For neutrinos from the muon decay one should substitute $m_{\pi}$ by $m_{\mu}$ and insert the lifetime of muon for $\tau_{0}$.) Notice that this ratio depends on the first power of the neutrino energy. Numerically, we find

$$
\frac{d_{L}}{\sigma_{x}} \sim 0.1\left(\frac{\Delta m^{2}}{8 \times 10^{-5} \mathrm{eV}^{2}}\right)\left(\frac{L}{100 \mathrm{Mpc}}\right)\left(\frac{10 \mathrm{TeV}}{E_{\nu}}\right)\left(\frac{3 \times 10^{-8} \mathrm{sec}}{\tau_{0}}\right) .
$$

This ratio shows that despite the extremely long baselines, coherence is in general maintained. This is always correct for neutrinos with $E_{\nu} \geq 1 \mathrm{TeV}$ originating from the muon decay (even if we take $L$ as large as the present size of the Universe). Only for relatively low energy neutrinos, $E_{\nu} \sim \mathrm{TeV}$, from the pion decay, the coherence can be lost.

\subsection{Interaction with particles in medium}

If the parent particle (pion, muon) scatters off the particles in the medium, the length of the wavepacket of the produced neutrino will be shorter than in the interaction-free case. Here, for illustrative purposes we consider a radiation dominated environment as expected in most models for the GRB sources. According to the fireball models [4, 15], neutrinos at the GRB sources are produced in relativistic jets which have boost factors $\left(\Gamma_{j e t}\right)$ of order of $10^{2}$ in the observer frame. In the frame comoving with the jet, which we will call the jet-frame, photons have a thermal distribution with a typical temperature of $T_{\gamma} \sim$ few $\mathrm{keV}$ [4]. In this environment, the main interaction process for the charged particle is scattering off the photons.

Let us consider the relevance of collisions and obtain the size of the neutrino wavepacket $\sigma_{x}^{j e t}$ in the jet-frame. The most energetic pions (and muons) are supposed to move in the direction of jet and therefore their energy in the jet frame can be estimated as

$$
E_{\pi(\mu)}^{j}=\frac{E_{\pi(\mu)}}{\Gamma_{j e t}} \approx 100 \mathrm{GeV}\left(\frac{E_{\pi(\mu)}}{10 \mathrm{TeV}}\right)\left(\frac{10^{2}}{\Gamma_{j e t}}\right),
$$

where $E_{\pi}\left(E_{\mu}\right)$ is the energy of the pion (the muon) in the observer frame.

The average distance that the parent particle travels between two successive collisions can be estimated as

$$
\ell_{\mathrm{col}} \sim \frac{1}{\sigma n_{\gamma}},
$$


where $n_{\gamma}=\left[2 \zeta(3) / \pi^{2}\right] T^{3}$ is the photon density and $\sigma$ is the total cross-section of the scattering. In the photon-pion center of mass system, the momentum of the pion is of order of $\sim E_{\pi}^{j} T_{\gamma} / m_{\pi} \sim 4 \mathrm{MeV} \ll m_{\pi}$; that is, in this frame the pion is non-relativistic. In this regime, the cross-section of the Compton scattering is given by the Thompson formula:

$$
\sigma=\frac{8 \pi \alpha^{2}}{3 m_{\pi}^{2}}
$$

In general, to calculate $\ell_{c o l}$, we should take into account the energy distribution of the photons in the thermal bath but since in this non-relativistic regime the cross-section is not sensitive to the momentum of the photon, the approximation we have made is justified. Inserting $n_{\gamma}$ and $\sigma$ in Eq. (27), we obtain

$$
\ell_{\text {col }} \sim 3 \times 10^{4} \mathrm{~cm}\left(\frac{4 \mathrm{keV}}{T_{\gamma}}\right)^{3} .
$$

In the case of muon scattering, $m_{\pi}$ has to be replaced by $m_{\mu}$. Notice that the mean free path is approximately the same for muons and pions.

In the jet-frame the pions are ultra-relativistic so the majority of the produced neutrinos are emitted within a narrow cone with an opening angle of $m_{\pi} / E_{\pi}^{j}$, oriented along the momentum of the parent particle. We will refer to this cone as "the emission cone." The energy transfer in each collision is negligible (i.e., the collisions are elastic) and only the momentum of the charged particle randomly changes direction by an angle of size $\sim T_{\gamma} / m_{\pi}$. The smallness of the momentum change in each collision (i.e., $T_{\gamma} / m_{\pi} \ll$ $\left.m_{\pi} / E_{\pi}^{j}\right)$ implies that if the direction of the momentum of the parent particle before collision is such that the line of sight lies within the emission cone, after the collision, the line of sight will still remain within the emission cone.

All the consideration we had so far in this sub-section holds equally for the pion and the muon. However, we should bear in mind that the neutrino production by the former takes place via a two-body decay while from the latter it takes place via a three-body decay. As a result, the widths of the neutrino wavepackets produced by them are different. In the following we first discuss the pion decay and we then consider the muon decay.

During the time between two successive collisions $\left(\ell_{c o l} / c\right)$, the pion emits 
a wavepacket whose size is of order of

$$
\sigma_{x, \pi}^{j e t} \sim \ell_{c o l} \frac{m_{\pi}}{E_{\pi}^{j}} \frac{E_{\nu}^{0}}{E_{\nu}^{j}}=\ell_{c o l} \frac{m_{\pi}}{E_{\pi}^{j}} \frac{m_{\pi}^{2}-m_{\mu}^{2}}{2 m_{\pi} E_{\nu}^{j}},
$$

where $E_{\nu}^{0}$ is the energy of the neutrino in the rest frame of the pion (see Eq. (18)). Notice that, in Eq. (29), we have taken into account the Lorentz contraction of the wavepacket as discussed in sec. 3.1 (see Eq. (20)).

After the collision, the momentum of the pion is changed by $\left|\Delta \vec{p}_{\pi}^{j}\right| \sim$ $T_{\gamma} E_{\pi}^{j} / m_{\pi}$ in a direction transverse to the momentum $\left(\Delta \vec{p}_{\pi}^{j} \cdot \vec{p}_{\pi}^{j} \ll\left|\Delta \vec{p}_{\pi}^{j}\right|\left|\vec{p}_{\pi}^{j}\right|\right)$. Writing the kinematics of scattering, we find that the difference between the energy of the neutrino emitted in our direction before and after the collision is $\sim T_{\gamma}\left(E_{\pi}^{j} / m_{\pi}\right)^{2}$ which is much larger than $\left(\sigma_{x, \pi}^{j e t}\right)^{-1}$ given in Eq. (29). This means that the wavepackets emitted before and after the collision are incoherent and the effective size of the wavepacket in the jet frame is therefore given by Eq. (29).

The spectrum of the neutrinos produced by the muon is continuous. Thus, even after a collision the wavepacket of the neutrino will have a component of the same energy as before. However, if we wait long enough the direction of the parent particle will change so much that the line of sight will exit the emission cone. After this time, the amplitude of the neutrino emitted in the observation direction is negligible. Thus, the size of the wavepacket is determined by the time, $\Delta t$, during which the line of sight exits the emission cone. Let us estimate $\Delta t$. As mentioned, after each collision the momentum of the parent particle changes direction randomly by an angle of size $T_{\gamma} / m_{\mu}$ so, after $N$ collisions, the total rotation angle on average amounts to $\sqrt{N} T_{\gamma} / m_{\mu}$. Thus, for $E_{\mu}^{j} T_{\gamma} \ll m_{\mu}^{2}$, after $N \sim\left(m_{\mu}^{2} / E_{\mu}^{j} T_{\gamma}\right)^{2}$ collisions, the direction of the momentum changes by an angle of $\sim m_{\mu} / E_{\mu}^{j}$ and the line of the sight therefore exits the emission cone. Since the time between two successive collisions is of order of $\ell_{c o l} / c$, we conclude that

$$
\Delta t \sim \frac{\ell_{c o l}}{c}\left(\frac{m_{\mu}^{2}}{E_{\mu}^{j} T_{\gamma}}\right)^{2} .
$$

Taking into account the Lorentz contraction (see sec. 3.1) for neutrinos emitted in the forward direction we obtain

$$
\sigma_{x, \mu}^{j e t} \sim c \Delta t \frac{m_{\mu}}{E_{\mu}^{j}} \frac{E^{0}}{E_{\nu}^{j}} \sim \frac{\ell_{c o l}}{3} \frac{m_{\mu}^{6}}{T_{\gamma}^{2}\left(E_{\nu}^{j}\right)^{2} E_{\mu}^{2}},
$$


where $E^{0}$ is the energy of the neutrino in the rest frame of the muon which is of order of $m_{\mu} / 3$. Notice that this relation is valid only if $E_{\mu}^{j} T_{\gamma} \ll m_{\mu}^{2}$. For larger values of $E_{\mu}^{j} T_{\gamma}$, a single collision will be enough for the line of sight to exit the emission cone (i.e., $N=1$ ) and therefore $\sigma_{x, \mu}^{j e t} \sim \ell_{c o l}\left(m_{\mu} E^{0}\right) /\left(E_{\mu}^{j} E_{\nu}^{j}\right)$.

As we discussed in the previous section, $\sigma_{E} / E$, or equivalently $E \sigma_{x}$, is invariant under the Lorentz transformations. Thus, from Eq. (29), we find that in the observer frame, the wavepackets of the neutrinos produced by the pion are of size of

$$
\sigma_{x, \pi}=\sigma_{x, \pi}^{j e t} \frac{E_{\nu}^{j e t}}{E_{\nu}} \sim 3 \times 10^{-5} \mathrm{~cm}\left(\frac{10 \mathrm{TeV}}{E_{\nu}}\right)^{2}\left(\frac{4 \mathrm{keV}}{T_{\gamma}}\right)^{3} \frac{\Gamma_{j e t}}{100} .
$$

With this wavepacket size, we obtain from Eq. (14) that for neutrinos produced by the pions, the wavepackets of all three neutrino mass eigenstates will be separated on their way to Earth from the GRB sources.

Similarly, using the invariance of $E \sigma_{x}$ we obtain

$$
\sigma_{x, \mu}=\sigma_{x, \mu}^{j e t} \frac{E_{\nu}^{j e t}}{E_{\nu}} \sim 5 \times 10^{-3} \mathrm{~cm}\left(\frac{4 \mathrm{keV}}{T_{\gamma}}\right)^{5}\left(\frac{10 \mathrm{TeV}}{E_{\nu}}\right)^{4}\left(\frac{\Gamma_{j e t}}{100}\right)^{3}
$$

From Eq. (14) we conclude that unlike the case of neutrinos from the pion decay, the different mass components of the neutrinos produced by the muon will keep overlapping until they reach the Earth. In other words, the coherence of the neutrinos from the muon decay is maintained.

In the energy-momentum space, the wavepacket sizes in Eqs. (31,32) translate into

$$
\frac{\sigma_{E, \pi}}{E_{\nu}} \sim 10^{-13}\left(\frac{E_{\nu}}{10 \mathrm{TeV}}\right)\left(\frac{T_{\gamma}}{4 \mathrm{keV}}\right)^{3}\left(\frac{100}{\Gamma_{j e t}}\right) \quad\left(\text { for } \quad \frac{E_{\nu} T_{\gamma}}{\Gamma_{j e t}} \ll m_{\pi}^{2}\right)
$$

and

$$
\frac{\sigma_{E, \mu}}{E_{\nu}} \sim 10^{-15}\left(\frac{E_{\nu}}{10 \mathrm{TeV}}\right)^{3}\left(\frac{T_{\gamma}}{4 \mathrm{keV}}\right)^{5}\left(\frac{100}{\Gamma_{j e t}}\right)^{3} \quad\left(\text { for } \frac{E_{\nu} T_{\gamma}}{\Gamma_{j e t}} \ll m_{\mu}^{2}\right) .
$$

Increasing the energy, $\sigma_{E}$ increases. Notice however that for $E_{\nu} T_{\gamma} / \Gamma_{j e t} \gtrsim m_{\pi}^{2}$, the above formulas are not valid for two reasons: (i) in this regime the cross section is not given by the Thompson formula; (ii) as discussed before, for 
high energies, Eq. (30) is not valid. Taking into account these considerations we find that

$$
\frac{\sigma_{E, \pi}}{E_{\nu}} \sim \frac{\sigma_{E, \mu}}{E_{\nu}} \sim 5 \times 10^{-12}\left(\frac{T_{\gamma}}{4 \mathrm{keV}}\right)^{3} \quad\left(\text { for } \quad \frac{\mathrm{E}_{\nu} \mathrm{T}_{\gamma}}{\Gamma_{\text {jet }}} \gtrsim \mathrm{m}_{\pi}^{2}\right) .
$$

\subsection{The effects of magnetic fields}

Let us discuss the effects of the magnetic field present in the source on the width of the neutrino wavepacket. The magnetic field at the GRB sources can be as large as $\sim 10^{9}$ Gauss $[4,5,6]$. For simplicity, we assume that the magnetic field is constant and uniform: $(\vec{B}=B \hat{z})$. Later on, we will show that this assumption is justified. Notice that although the magnetic fields in the sources under consideration are quite large but still $e B \ll E^{2}$ and we can therefore treat the effects of the magnetic field classically. That is, we can describe the state of the charged particle as a plane wave whose momentum in the direction transverse to the magnetic field slowly rotates in time. As we will see, the decays of the pion and muon have to be treated differently.

Let us first discuss the pion decay. In the magnetic field, the charged pions move on circular (spiral) trajectories with Larmor radius $R=E_{\pi} / e B$. The energy of the emitted neutrino in a two body decay depends on the angle between its momentum and the momentum of the pion which, in turn, is a function of time. That is, the energy of the emitted neutrino at time $t, E_{\nu}(t)$, differs from that at time $t+\Delta t$ :

$$
\Delta E_{\nu} \equiv E_{\nu}(t+\Delta t)-E_{\nu}(t) .
$$

Taking into account the Lorentz contraction (20) we find that the length of the neutrino wavepacket in the configuration space emitted during the period $\Delta t$ from a pion with energy of $E_{\pi}$ is given by

$$
\sigma_{x}=(c \Delta t) \frac{m_{\pi}}{E_{\pi}} \frac{E_{\nu}^{0}}{E_{\nu}}
$$

where $E_{\nu}^{0}$ is the energy of the neutrino in the rest frame of the pion (see Eq. (18)). Consider segments of the pion trajectory for which $\Delta E_{\nu}$ defined in Eq. (36) coincides with $\sigma_{E}=\sigma_{x}^{-1}$ :

$$
\sigma_{E}=\Delta E_{\nu}=\frac{1}{\Delta t}\left(\frac{E_{\pi}}{m_{\pi}}\right)\left(\frac{E_{\nu}}{E_{\nu}^{0}}\right)=\frac{1}{\Delta t}\left(\frac{2 E_{\pi} E_{\nu}}{m_{\pi}^{2}-m_{\mu}^{2}}\right) .
$$


The wavepackets emitted during successive $\Delta t$ can be resolved by a hypothetical detector with energy resolution $\sigma_{E}$ and time resolution $\sigma_{E}^{-1}$, so their effects sum up incoherently. Consequently, the coherence length of the neutrino wavepacket is determined by $\sigma_{E}^{-1}$ which can, in turn, be derived by solving Eq. (37). For this, one needs to determine $\Delta E_{\nu}$ or $E_{\nu}$ as a function of time.

The four-momentum of the pion can be written as

$$
\left(E_{\pi}, \quad p_{\pi} \sin \theta_{\pi} \cos \Phi(t), \quad p_{\pi} \sin \theta_{\pi} \sin \Phi(t), \quad p_{\pi} \cos \theta_{\pi}\right),
$$

where $\Phi(t)$ is the rotation phase:

$$
\Phi(t)=\frac{e B\left(t-t_{0}\right)}{E_{\pi}} .
$$

Notice that $E_{\pi}$ is constant. Let us denote the unit vector in the direction from the source to the detector by $\hat{l}$, and define the axis $\hat{y}$ as $\hat{y}=\hat{l} \times \hat{z} /|\hat{l} \times \hat{z}|$. In this frame, the neutrino emitted at time $t$ towards the detector has the following four-momentum

$$
E_{\nu}(t)\left(1, \sin \theta_{\nu}, 0, \cos \theta_{\nu}\right),
$$

where $\cos \theta_{\nu}=(\hat{l} \cdot \hat{z})$ and

$$
E_{\nu}(t)=\frac{m_{\pi}^{2}-m_{\mu}^{2}}{2\left[E_{\pi}-p_{\pi} \cos \left(\theta_{\nu}-\theta_{\pi}\right)+2 p_{\pi} \sin \theta_{\pi} \sin \theta_{\nu} \sin ^{2} \frac{\Phi(t)}{2}\right]} .
$$

Notice that due to the two-body character of the pion decay, the neutrino energy in the rest frame of the pion is fixed, and in the observer frame it is uniquely determined by the angle between the momenta of the pion and the neutrino. From Eq. (41), we obtain

$$
\Delta E_{\nu}=\frac{2 e B E_{\nu}^{2} \sin \theta_{\nu} \sin \theta_{\pi} \sin \Phi}{m_{\pi}^{2}-m_{\mu}^{2}} \Delta t .
$$

Only a small fraction of the neutrinos (of order of $\left.\left(m_{\pi} / p_{\pi}\right)^{2}\right)$ are emitted in directions for which the angle between the neutrino and pion momenta is larger than $m_{\pi} / p_{\pi}$. We therefore concentrate on the angles and emission time for which the line of sight lies inside the emission cone:

$$
\left(\theta_{\nu}-\theta_{\pi}\right) \sim \sin \Phi(t) \lesssim m_{\pi} / p_{\pi} \ll 1 .
$$


For such values of $t, \theta_{\pi}$ and $\theta_{\nu}$, from Eqs. $(37,42)$ we obtain

$$
\sigma_{E} \sim\left[\frac{4 e B E_{\nu}^{3} m_{\pi} \sin \theta_{\pi} \sin \theta_{\nu}}{\left(m_{\pi}^{2}-m_{\mu}^{2}\right)^{2}}\right]^{1 / 2}
$$

Let us apply these results to neutrinos which are produced in relativistic jets $[4,15]$. We perform our analysis in the (comoving) jet-frame where the electromagnetic field is predominantly composed of the magnetic component 5. Using the invariance of $\sigma_{E} / E$ (see Eq. (19)), we can immediately write the width in the observer frame, $\sigma_{E}$, in terms of the energy width in the jet-frame, $\sigma_{E}^{j e t}$ :

$$
\frac{\sigma_{E}}{E_{\nu}}=\frac{\sigma_{E}^{j e t}}{E^{j e t}} \sim \sqrt{\frac{e B E_{\nu} m_{\pi}}{\Gamma_{j e t}\left(m_{\pi}^{2}-m_{\mu}^{2}\right)^{2}}} \sim 10^{-4}\left(\frac{100}{\Gamma_{j e t}} \frac{E_{\nu}}{10 \mathrm{TeV}} \frac{B}{10^{7} \mathrm{Gauss}}\right)^{1 / 2},
$$

where we have taken $4 \sin \theta_{\pi} \sin \theta_{\nu} \sim 1$ and $E_{\nu}^{j e t} \sim E_{\nu} / \Gamma_{j e t}$. The width of the wavepacket in the configuration space is given by

$$
\sigma_{x} \sim \sigma_{E}^{-1} \sim 2 \times 10^{-14} \mathrm{~cm}\left(\frac{\Gamma_{j e t}}{100} \frac{10^{7} \mathrm{Gauss}}{B}\right)^{1 / 2}\left(\frac{10 \mathrm{TeV}}{E_{\nu}}\right)^{3 / 2} .
$$

Conferring the estimations in Eqs. (46) and (31) we conclude that for $B>$ $10^{-11}$ Gauss, interaction of pions with the magnetic field is more effective in shortening the wavepacket than their scattering off the particles in the medium. That is, in practice, the size of the wavepacket of neutrinos produced in a cosmic neutrino source is given by Eq. (46) rather than by Eq. (31).

Using the ratio in Eq. (45) and the condition shown in Eq. (8), we find that the loss of coherence over cosmological distances occurs if

$$
B \gg 5 \times 10^{-13} \text { Gauss }\left(\frac{8 \times 10^{-5} \mathrm{eV}^{2}}{\Delta m^{2}}\right)^{2}\left(\frac{100 \mathrm{Mpc}}{L}\right)^{2} \frac{E_{\nu}}{10 \mathrm{TeV}} \frac{\Gamma_{j e t}}{100} .
$$

Let us now consider neutrinos from the muon decay. Due to the threebody character of the decay, the energy spectrum of the neutrino emitted in a given direction is continuous. Thus, the consideration that we had in

\footnotetext{
${ }^{5}$ In the observer frame, according to the Lorentz transformation in addition to the magnetic field, an electric field will be also present.
} 
the case of the two-body decay of the pion does not apply here. Since the muon is ultrarelativistic, the majority of the emitted neutrinos are oriented in the direction of the muon momentum within the emission cone. Only a small fraction of $O\left(m_{\mu}^{2} / E_{\mu}^{2}\right)$ is emitted outside the emission cone. Since the momentum of the muon rapidly rotates, only for a short period, $\Delta t$, the line of sight lies inside the emission cone. A reasonable estimate for $\Delta t$ is the time interval during which the angle between the line of sight and the momentum of the muon is smaller than $m_{\mu} / E_{\mu}:\left(e B \Delta t / E_{\mu}\right) \lesssim m_{\mu} / E_{\mu}$. We then find that in the jet-frame

$$
\sigma_{E}^{j e t}=(\Delta t)^{-1}\left(\frac{E_{\mu}^{j e t}}{m_{\mu}}\right)^{2}=\frac{e B}{m_{\mu}}\left(\frac{3 E_{\nu}^{j e t}}{m_{\mu}}\right)^{2},
$$

where we have taken $E_{\nu} \sim E_{\mu} / 3$. Using the invariance of $\sigma_{E} / E$, we obtain

$$
\frac{\sigma_{E}}{E_{\nu}}=\frac{\sigma_{E}^{j e t}}{E_{\nu}^{j e t}}=5 \times 10^{-8} \frac{B}{10^{7} \text { Gauss }} \frac{E_{\nu}}{10 \mathrm{TeV}} \frac{100}{\Gamma_{j e t}},
$$

where $E_{\nu}$ is the energy in the observer frame: $E_{\nu}=E_{\nu}^{j e t} / \Gamma_{j e t}$. Thus, in the observer frame, the length of the wavepacket is

$$
\sigma_{x} \sim \sigma_{E}^{-1} \sim 5 \times 10^{-11} \mathrm{~cm}\left(\frac{10 \mathrm{TeV}}{E_{\nu}}\right)^{2} \frac{10^{7} \text { Gauss }}{B} \frac{\Gamma_{j e t}}{100} .
$$

Again, comparing the estimations in Eq. (50) and in Eq. (32) we find that for $B>10^{-1}$ Gauss, the interaction of muons with the magnetic field is more effective in shortening the wavepacket size than their scattering off the particles in the medium. In the presence of the magnetic field, the wavepacket size is therefore given by Eq. (50) rather than by Eq. (32).

To obtain complete loss of coherence (see, Eq. (8)), the following condition has to be fulfilled:

$$
B \gg 10 \text { Gauss } \frac{100 \mathrm{Mpc}}{L} \frac{8 \times 10^{-5} \mathrm{eV}^{2}}{\Delta m^{2}} \frac{\Gamma_{j e t}}{100} .
$$

Notice that while in the case of the pion decay, $\sigma_{E}$ is proportional to $B^{1 / 2}$ (see Eq. (45)), in the muon decay case, the dependence on $B$ is linear (see Eq. (49)). This explains the huge difference between the lower limits on $B$ shown in Eqs. $(47,51)$. 
In the analysis, we have taken the magnetic field to be constant. In fact, this assumption is justified as long as the variation of the magnetic field over scales of $c \Delta t$ [where $\Delta t$ is the time segment that determines $\sigma_{E}$; see Eqs. $(37,48)]$ is negligible. Taking $E_{\nu}=10 \mathrm{TeV}$ and $\Gamma_{j e t}=100$, we find that for the case of pions, $c \Delta t \sim 10^{-5} \mathrm{~cm}\left(10^{7} \mathrm{Gauss} / B\right)^{1 / 2}$ and for the case of muon, $c \Delta t \sim 4 \times 10^{-3} \mathrm{~cm}\left(10^{7}\right.$ Gauss $\left./ B\right)$. Notice that these values of $c \Delta t$ are much smaller than the jet size [4] which justifies taking the magnetic field constant.

Notice that according to Eqs. $(45,49)$, the ratio $\sigma_{E} / E_{\nu}$ increases with energy. Thus, in the framework of the quasi-degenerate mass scheme, for very high energies and magnetic fields (i.e., for $E_{\nu} \gg 10 \mathrm{TeV}$ and $B \gtrsim 10^{9}$ Gauss), the spread of the wavepacket can overcome the wavepacket separation (see Eq. (16)).

Our approximation of taking the momentum of the particle constant over $\Delta t$ is valid only as long as $\Delta t E_{\nu} \gg 1$. It is straightforward to check that for

$$
\left(\frac{B}{10^{7} \text { Gauss }}\right)\left(\frac{E_{\nu}}{10 \mathrm{TeV}}\right)\left(\frac{100}{\Gamma_{j e t}}\right)>10^{8},
$$

this condition is not fulfilled, so Eqs. $(45,49)$ which were derived on the basis of this approximation are not valid. For

$$
\left(\frac{B}{10^{7} \text { Gauss }}\right)\left(\frac{E_{\nu}}{10 \mathrm{TeV}}\right)\left(\frac{100}{\Gamma_{j e t}}\right) \sim 10^{7},
$$

in both muon and pion decay cases, $\sigma_{E} / E_{\nu} \sim 0.1$ and the coherent correction to the spectrum can be significant. We will discuss this point in section 4.4.

\section{Applications}

\subsection{Measuring the size of the wavepakets}

As we discussed in the previous sections the sizes of the neutrino wavepackets carry important information on conditions of neutrino production: on strength of the magnetic field, on radiation and matter density, on distance to the source. The sizes of the packets differ for neutrinos from the muon and pion decays. Can the sizes of the wavepackets of cosmic neutrinos be measured? Are they relevant for observations? 
In Ref. [14], it has been shown that the density matrix of a "stationary" beam of particles is entirely determined by the energy spectrum and does not depend on the sizes of the wavepackets. As a result, all the "stationary" beams composed of wavepackets of different sizes are equivalent and cannot be distinguished by measurement provided that they have the same energy spectra. However, in reality, even a macroscopically stationary beam composed of wavepackets of finite size is not stationary at the microscopic level. As pointed out in [14], for a non-stationary beam, using time information in principle makes determining the size of the constituent wavepackets a possibility.

Consider a burst of neutrinos emitted from a source at distance of $L$ during time interval $t_{b}$. If the emission is spherically symmetric, at the time of detection neutrinos will occupy a shell with a volume of $4 \pi L^{2} t_{b}$. The total number of emitted neutrinos is $E_{\text {burst }} / E_{\nu}$, where $E_{\text {burst }}$ is the total energy of the burst and $E_{\nu}$ is the average energy of neutrinos. The average time between two successive neutrinos passing through an area of $S$ (perpendicular to the line of sight of the source) is

$$
\begin{gathered}
\Delta t=\left(\frac{4 \pi L^{2}}{S}\right)\left(\frac{E_{\nu}}{E_{\text {burst }}}\right) t_{b} \\
\sim(1 \mathrm{sec})\left(\frac{L}{100 \mathrm{Mpc}}\right)\left(\frac{1 \mathrm{~m}^{2}}{S}\right)\left(\frac{E_{\nu}}{10 \mathrm{TeV}}\right)\left(\frac{10^{52} \mathrm{erg}}{E_{\text {burst }}}\right)\left(\frac{t_{b}}{1 \mathrm{sec}}\right) .
\end{gathered}
$$

The time resolution of detector can be much better than $\Delta t=1 \mathrm{sec}$. As a result, the flux will appear as non-stationary at the microscopic scale. Suppose information on the arrival time of each wavepacket is provided. Then, by recording the detection time with precision better than $\Delta t$, one can in principle extract information on the size of wavepackets composing such a flux. If a detection takes place at time $\tau$ from the arrival time of the "center" of a certain wavepacket, the wavepacket size has to be larger than $c \tau$. The question is whether there is any possibility to obtain the arrival time of each wavepacket. The answer is positive if, for example, the injection (production of pions) has some fine structure with $\delta t<\sigma_{x} \ll \Delta t$, associated to emission of the short $\gamma$ - burst or gravitational waves; i.e., in this case, the arrival time of the "center" (or initial moment) of the neutrino wavepacket can be determined from the arrival time of $\gamma$ 's (or gravitational waves). Unfortunately, present models do not predict such strict time correlation $(\delta t \ll \Delta t)$ 
between the $\gamma$ and $\nu$ bursts. In more realistic situations when no information on spatial distribution of the wavepackets within the duration of the burst is available, even recording the detection time will not help us to determine the wavepacket size.

The present situation argument is in line with the theorem proved in sec. D.3 of [13] which claims that for any flux composed of "short" wavepackets with a certain spatial distribution, it is possible to find an equivalent flux (i.e., having the same density matrix) composed of long wavepackets with width $\Delta z \gg \delta z$ provided that the long wavepackets cover the same part of the space in which the short wavepackets are distributed. Although the sizes of the wavepackets cannot be practically determined, their effects can appear in the energy spectrum through the coherent broadening phenomenon that is discussed in sec. 4.4.

\subsection{Coherence of neutrinos from various cosmic sources}

In this section, we check if the condition of coherence loss can be satisfied for various sources of high energy neutrinos. The most powerful sources of neutrinos (Gamma Ray Burst sources and AGNs) are located at very far distances $(L>1 \mathrm{Mpc})$. From Eqs. (47,51), and for known values of $\Delta m^{2}$ we observe that even a relatively small magnetic field is enough to cause loss of coherence over such distances. The magnetic fields in the GRB sources [4] are of course larger than these bounds. Notice that the bound shown in Eq. (51) is independent of the energy while the bound in Eq. (47) becomes stronger with energy. Taking $E_{\nu}$ as high as $10^{21} \mathrm{eV}$, from (47) we conclude that $B$ larger than 0.1 milli-Gauss at the source is enough to destroy the coherence of the neutrinos from the pion decay over cosmological distances.

Now let us discuss the cosmogenic neutrinos. Even though the baseline for these neutrinos is of cosmological scale, the coherence of the neutrinos produced by the muon decay is not lost because in the intergalactic area, where these neutrinos are produced, the magnetic field is too weak to satisfy Eq. (51). However, it is not out of question to have an intergalactic magnetic field large enough to satisfy Eq. (47) for $E_{\nu}<\mathrm{EeV}$, and cause loss of coherence for the cosmogenic neutrino from the pion decay [16].

For neutrinos arriving from the center of galaxy $(L \sim 10 \mathrm{kpc})$, to satisfy 
Eq. (47) the magnetic field at the source has to be larger than $5 \times 10^{-5}$ Gauss while to satisfy Eq. (51) the value of the magnetic field has to be larger than $10^{5}$ Gauss. If the magnetic field is between $5 \times 10^{-5}$ Gauss and $10^{5}$ Gauss, the part of the flux from the pion decay will lose its coherence but the other part from the muon decay will maintain its coherence.

For "solar atmospheric neutrinos" (i.e., neutrinos produced via interaction of the cosmic ray particles with the atmosphere of the sun [18]), neither of the bounds in Eq. (47) and Eq. (51) is satisfied: Setting $L=5 \times 10^{-6} \mathrm{pc}$ (the distance between the Sun and the Earth), $\Gamma_{j e t}=1, \Delta m^{2}=\Delta m_{\text {atm }}^{2}$ and $E_{\nu} \sim 1 \mathrm{GeV}$, we find that in order to satisfy Eqs. (47) and (51) the magnetic field at the solar surface has to be larger than $10^{5}$ Gauss and $10^{11}$ Gauss, respectively; that is while, the magnetic field on the surface of the sun is less than $10^{4}$ Gauss so the coherence is not destroyed.

\subsection{Coherence of neutrinos from terrestrial sources}

In this section, for comparison we discuss the coherence in terrestrial long baseline experiments with a strong magnetic field at the production site. Taking $E_{\nu} \sim 10 \mathrm{GeV}, L \sim 2 R_{\oplus}, \Gamma_{\text {jet }}=1$ and $\Delta m^{2}=2 \times 10^{-3} \mathrm{eV}^{2}$, from Eq. (47) we find that the magnetic field at the source has to be larger than $10^{9}$ Tesla to obtain complete loss of coherence. For $\Delta m^{2} \sim 1 \mathrm{eV}^{2}$ or larger (as suggested by LSND; see [17] for recent status of such models), a magnetic field of 1000 Tesla at the source would be required to cause loss of coherence over a baseline of the Earth size. From Eq. (45), for $E_{\nu} \sim 30 \mathrm{GeV}, \Gamma_{\text {jet }}=1$ and $B \sim 1$ Tesla, we obtain $\sigma_{E} / E_{\nu} \sim 10^{-6}$. In practice, the energy resolution of the detector will be larger (worse) than this value.

For a setup such as the muon storage ring of the neutrino factory the magnetic field is significant only at rounded edges of the rectangular track of the muons. The neutrinos reaching the detector are produced while the muon propagates through the side of the track that is aligned towards the detector. The length of the wavepacket of neutrino is therefore determined by the length of this side of the track, $l_{s t r}$. Taking into account Lorentz contraction ( see sec 3.1) we find that the length of the wavepacket emitted in the direction of the muon momentum is equal to

$$
\sigma_{x}=l_{s t r} \frac{m_{\mu}^{2}}{E_{\mu}^{2}} .
$$


Taking $E_{\mu}=30 \mathrm{GeV}$ and $l_{\text {str }}=600 \mathrm{~m}$, we obtain $\sigma_{x}=0.7 \mathrm{~cm}$. From Eq. (14), we find that to break the coherence for the atmospheric mass splitting the baseline has to be larger than $\sim 0.1 \mathrm{Mpc}$. Obviously, the coherence of the neutrinos reaching the detector of the neutrino factory will be maintained.

\subsection{Effects of coherent broadening}

Coherent broadening can lead to deformation of the energy spectrum. A well-known example is the coherent broadening of a monochromatic line in atomic spectroscopy. The effects smooth down any sharp structure of scale smaller than $\sigma_{E}$ in the spectrum. If the coherent broadening is symmetric (i.e., if the broadening takes "a line" of energy $E$ to $\left.\left(E-\sigma_{E} / 2, E+\sigma_{E} / 2\right)\right)$, the leading order correction to the spectrum $(F)$ will be given by

$$
F \rightarrow\left[F+\frac{\sigma_{E}^{2}}{2} \frac{d^{2} F}{d E^{2}}\right] \frac{1}{N}
$$

where $N$ is a normalization factor. However, if the broadening is asymmetric, i.e., if $E$ is mapped onto $\left(E-\sigma_{E}(1 / 2+a), E+\sigma_{E}(1 / 2-a)\right)$ with $a \neq 0$, the first order correction to the spectrum will be larger and linear in $\sigma_{E}$, i.e.,

$$
F \rightarrow\left(F+\frac{d F}{d E} \sigma_{E} a\right)
$$

In the conservative case of symmetric broadening, the effects on spectrum can be significant if $\sigma_{E} \sim\left[\left(d^{2} F / d E^{2}\right) / F\right]^{-1 / 2}$, where $F$ is the spectrum without taking into account the coherent effects. Notice that the effect takes place at production, so the distance between the source and detector does not play any role in this consideration. For cosmic neutrinos, the spectrum is predicted to follow a power law behavior: $F \propto E^{-n}$ with $n \simeq 2$. For such a spectrum, the coherent broadening can be significant if $\sigma_{E} / E_{\nu} \sim 0.1$. From Eqs. $(33,34,35)$, we observe that the coherent broadening due to scattering is completely negligible. However, as discussed in section 3.3, for the decay in the magnetic field $B \sim 10^{9} \operatorname{Gauss}_{j e t}\left(10 \mathrm{PeV} / E_{\nu}\right)$, the ratio $\sigma_{E} / E$ can be of order of 0.1 (see Eqs. $(45,49)$ ). For lower values of the magnetic field and energy, the coherent broadening is irrelevant. As discussed in [6], in mildly relativistic jets with $\Gamma_{j e t} \sim 3$ present in type Ib/c supernovae, the magnetic 
field can be as high as $10^{9}$ Gauss. In such an environment, the corrections to the spectrum due to the coherent broadening can be significant if $E_{\nu}>$ few $10 \mathrm{PeV}$. In principle, the energy loss through synchrotron radiation can hamper production of such high energy neutrinos. Notice, however that as shown in [26] through the same mechanism that protons are accelerated in the magnetic field, muons and charged mesons can also be accelerated. As a result, reaching energies above $\mathrm{PeV}$ is possible [26]. Since the dependence of $\sigma_{E}$ on energy is non-linear (see Eqs. $(45,49)$ ), the coherent effects can change the shape of the energy spectrum.

\subsection{Effects of incomplete averaging}

As we discussed in sec. 4.2, in some special cases, the coherence of cosmic neutrinos will be maintained. In case that coherence is not lost, the probability of neutrino oscillation can be represented as

$$
P=\bar{P}+P_{\text {int }}
$$

where $\bar{P}$ is the average probability and $P_{\text {int }}$ is the interference term which in the two neutrino scheme can be written as

$$
P_{\text {int }}=D \cos \phi(E, L)
$$

$\bar{P}$ and $D$ are independent of the energy of the neutrinos, $E$, and the baseline, $L$. The phase of oscillations in vacuum is defined in Eq. (5). In the case of three neutrino oscillation, there will be three interference terms associated to three different $\Delta m^{2}$.

The oscillatory terms are in practice averaged to zero for the following three reasons: (i) the integration of signal over the energy because of the finite energy resolution of the detector; (ii) the integration over the region of the production; and (iii) the summation over different cosmic sources. The third reason is specially inevitable. For example, from a single gamma burst source at $z \sim 1$, we expect only $10^{-1}-10$ events at a $1 \mathrm{~km}^{3}$ scale detector [4]. Fortunately, about $\sim 1000$ GRB are observed every year which can yield at least around a few hundred neutrino events per year at ICECUBE. When we combine the data from different GRB sources, averaging over baselines takes place. 
For typical values of the cosmic neutrino parameters, we obtain the phase

$$
\phi=6 \times 10^{13}\left(\frac{\Delta m^{2}}{8 \times 10^{-5} \mathrm{eV}^{2}}\right)\left(\frac{L}{100 \mathrm{Mpc}}\right)\left(\frac{10 \mathrm{TeV}}{E}\right) .
$$

Considering the fact that the phases are very large, $\phi \gg 1$, we expect the oscillatory term to average to zero. In the following, we discuss the error from neglecting these terms. We discuss the procedure of averaging for two neutrino mixing where only one interference term exists. Generalization of our results to the three neutrino case is straightforward. For simplicity and definiteness, we will consider averaging due to integration over the neutrino energy. Integration over source volume and summation over different sources do not produce new features and can be considered in a similar way. The "average" number of events of different types in the detector can be written as

$$
\langle N\rangle \simeq \int_{E_{t h}} d E \frac{d N}{d E}
$$

where $E_{t h}$ is the detection threshold and

$$
\frac{d N}{d E}=\frac{d N^{0}(E)}{d E} P(E, L)
$$

Here $d N^{0}(E) / d E$ gives the average number of events with neutrino energy $E$ which would be observed if there was no oscillation [i.e., if $P\left(\nu_{\alpha} \rightarrow \nu_{\beta}\right)=$ $\left.\delta_{\alpha, \beta}\right]$.

Let us divide the whole detectable energy range into very small intervals $\Delta E\left(\ll \Delta E_{L}\right)$ over which the dependence of $d N / d E$ on energy can be neglected. The contribution of each interval to the total number is given by a Poisson distribution whose average and variance are both determined by $(d N / d E) \Delta E$. According to the "central limit theorem" [19], the distribution of the total number is given by a Gaussian whose mean and variance $\left(\sigma^{2}\right)$ are both given by a sum (an integral) over $(d N / d E)$ :

$$
\left\langle(N-\langle N\rangle)^{2}\right\rangle=\langle N\rangle
$$

where $\langle N\rangle$ is given by Eq. (57). Because of the uncertainty in the measurement of $L$, we cannot in practice determine the value of $\cos \phi(E, L)$ and therefore that of $d N / d E$. However, regardless of the value of $\phi(E, L)$, the 
integral of the oscillatory terms (see Eq. (57)) vanishes up to a small threshold correction: $O\left[\left.\Delta E_{L}(d N / d E)\right|_{t h}\right] \ll \sqrt{N}$. Thus, in the analysis of cosmic neutrinos, we can safely drop the oscillatory terms.

As shown in [13], from the observational point of view, the effect of averaging of oscillation is equivalent to the effect of coherence loss when signals from separated wavepackets sum up in the detector. As discussed, the variance of the distribution of the number of observed events is also the same for both cases and is determined by the root of the number of events regardless of the shape of the neutrino spectrum.

\subsection{Kinematical decoherence versus quantum decoher- ence}

Throughout this paper, we have assumed that the evolution of the neutrino states from the source to the Earth is given by the standard quantum mechanics. As is well-known, within the quantum mechanics, a pure state remains pure forever. Quantum gravity is expected to modify the evolution of the quantum states $[20,21]$. As long as the modifications preserve unitarity and the average energy and moreover respect the second law of thermodynamics, the damping factors appear only in front of the oscillatory terms [22, 23]:

$$
\begin{gathered}
P\left(\bar{\nu}_{\alpha} \rightarrow \bar{\nu}_{\beta}\right) \simeq P\left(\nu_{\alpha} \rightarrow \nu_{\beta}\right) \simeq\left|U_{\alpha 1}\right|^{2}\left|U_{\beta 1}\right|^{2}+\left|U_{\alpha 2}\right|^{2}\left|U_{\beta 2}\right|^{2}+\left|U_{\alpha 3}\right|^{2}\left|U_{\beta 3}\right|^{2} \\
+2 \operatorname{Re}\left[U_{\beta 1}^{*} U_{\alpha 1} U_{\beta 2} U_{\alpha 2}^{*}\right] \cos \Delta_{12} e^{-L \gamma_{12}}+2 \operatorname{Re}\left[U_{\beta 1}^{*} U_{\alpha 1} U_{\beta 3} U_{\alpha 3}^{*}\right] \cos \Delta_{13} e^{-L \gamma_{13}} \\
+2 \operatorname{Re}\left[U_{\beta 2}^{*} U_{\alpha 2} U_{\beta 3} U_{\alpha 3}^{*}\right] \cos \Delta_{32} e^{-L \gamma_{32}}
\end{gathered}
$$

where $\Delta_{i j}=\Delta m_{i j}^{2} L /\left(2 E_{\nu}\right)$. Thus, in the case of the cosmic neutrinos, for which either the interference terms disappear or their effects average to zero, the new damping factors are irrelevant. To have a deviation from the standard picture some of the assumptions that went into this conclusion (e.g., conservation of the average energy) have to be relaxed [24]. Our results are in accord with [25].

\section{Conclusions and Summary}

In this paper, we have explored various aspects of the coherence of the cosmic neutrinos with special emphasis on the neutrino flux in the (1-100) TeV 
energy range. Since the three neutrino mass eigenstates composing a single flavor state have different velocities, they spatially separate on their way to the detectors (see Eqs. $(8,12)$ ) provided that the source is far enough. Their effects in a detector cease to interfere and the oscillatory behavior of the probabilities disappears. The degree of separation depends on the size of the wavepacket, which in turn, is determined by the properties of the environment at the source.

We have evaluated the length of the neutrino wavepackets produced by the pion and muon in various environments. In an interaction-free medium, the wavepacket sizes in the observer frame from both the pion and muon decay are determined by the lifetime of the parent particle, the energy of the parent particle and direction of neutrino with respect to the momentum of the decaying particle. The size of the wavepacket does not depend on the feature of the decay (two-body vs. three-body decay). In contrast, when the parent particles undergo interactions before decay, the wavepacket sizes become dramatically sensitive to the shape of spectrum. That is despite the fact that the electromagnetic interactions of the muon and pion are practically the same, the neutrino wavepacket sizes and their dependence on the parameters become completely different for the pion and muon cases.

We have found that in an interaction-free environment, the wavepacket length is large enough to maintain coherence even for neutrinos traveling over cosmological distances. So the high energy cosmogenic neutrinos should arrive in coherent states

Scattering of the parent charged particle off the particles present in the medium decreases the length of the produced neutrino wavepackets. We have evaluated the amount of shortening of the wavepacket in a radiation dominated environment such as the one described in [4] as the GRB source. We have found that in the case of neutrinos produced by the pion, scattering can cause complete loss of coherence (even for $\Delta m_{\text {sol }}^{2}$ ) over cosmological distances. However, in the case of neutrinos produced by the muon with $E_{\nu} \lesssim 100 \mathrm{TeV}$, the effects of scattering are too small to cause coherence loss. In contrast, we have shown that even a tiny magnetic field at the production region can considerably widen the width of the neutrino wavepacket. A magnetic field as low as a few ten Gauss at the source is enough for effective loss of coherence (see Eqs. $(47,51)$ ). Based on the above consideration, we have concluded that because of the sizeable magnetic fields, all the neutrinos from 
remote point sources such as GRBs and AGNs lose their coherence before reaching the Earth. We have found that the dependence of the wavepacket size on the magnetic field and energy is quite different for neutrinos from the pion and muon decay. As a result, it is conceivable to have a situation that neutrinos from pion decay lose their coherence but those from the muon decay maintain theirs. Such a situation can be realized for cosmogenic neutrinos as well as for "solar atmospheric neutrinos". For all realistic terrestrial setups the coherence is maintained.

We have found that the coherent broadening can be important for

$$
B \sim 10^{9} \text { Gauss } \Gamma_{j e t}\left(10 \mathrm{PeV} / E_{\nu}\right) .
$$

Although such condition seems quite extreme, its realization is not out of question $[6,26]$. The dependence of $\sigma_{E}$ on energy is non-linear [see Eqs. $\left.(45,49)\right]$. As a result, the coherent effects can change the shape of the energy spectrum of cosmic neutrinos.

We have studied the case in which the coherence is maintained on the way from the source to detector. We have found that the variance of the distribution of the number of events is the same as the variance that we would expect in the absence of the oscillatory terms. As a result, neglecting the oscillatory terms does not induce a new source of error in determination of the neutrino parameters.

We have also discussed the effects of a deviation of the evolution of neutrino states from that in the standard quantum mechanics as one would expect in the framework of the quantum gravity. We have shown that as long as the deviation preserves unitarity and the average energy and moreover respects the second law of thermodynamics, the neutrino flavor composition at the detector is the same as the one without the deviation. To obtain a different composition, some of these assumptions have to be relaxed.

The neutrino pattern at the detector turns out to be the same for all three cases that we have studied in the present paper (i.e., maintenance and loss of coherence and a deviation from standard quantum mechanics) and as a result, these three cases cannot be distinguished by observation. Other possible applications of the results will be considered elsewhere. 


\section{Appendix}

In the following, we study the condition for the coherence loss in the energymomentum space. Let us consider a neutrino field of flavor $\alpha$ characterized by the following wavepacket

$$
\left|\nu_{\alpha}\right\rangle=\int f(\vec{p})|\alpha ; \vec{p}\rangle d^{3} p
$$

where $f(\vec{p})$ is a function of the momentum which is nonzero only in a small interval with width $\sigma_{p}$. The amplitude of finding the neutrino with flavor $\beta$, $\nu_{\beta}$, at point $\vec{x}$ and at time $t$ is

$$
\left\langle\nu_{\beta} ; \vec{x}, t \mid \nu_{\alpha}\right\rangle=\int \sum_{i} U_{\beta i}^{*} U_{\alpha i} e^{i\left[t\left(p+m_{i}^{2} /(2 p)\right)-\vec{x} \cdot \vec{p}\right]} f(\vec{p}) d^{3} p
$$

where $p \equiv|\vec{p}|$. Let us denote the spatial resolution by $\Delta X_{D}$. The probability of finding $\nu_{\beta}$ in a volume of $V=\left(\Delta X_{D}\right)^{3}$ at time $t=L$ (where $L$ is the distance between the source and the detector) is

$$
P\left(\nu_{\alpha} \rightarrow \nu_{\beta}\right)=\int_{V}\left|\left\langle\nu_{\beta} ; \vec{x}, L \mid \nu_{\alpha}\right\rangle\right|^{2} d^{3} x
$$

For any realistic detector, $\Delta X_{D}$ is far larger than the size of the wavepacket. As a result, we can replace the integration over $x$ with a delta function:

$$
P\left(\nu_{\alpha} \rightarrow \nu_{\beta}\right)=\int \sum_{i, j} U_{\beta i}^{*} U_{\alpha i} U_{\beta j} U_{\alpha j}^{*} e^{i\left(m_{i}^{2}-m_{j}^{2}\right)(L / 2 p)}|f(\vec{p})|^{2} d^{3} p .
$$

$f(\vec{p})$ is a relatively smooth function over an interval of size $\sigma_{p}$. For $\left(m_{j}^{2}-\right.$

$m_{i}^{2}$ ) satisfying the condition of loss of coherence (8), the integration of the interference terms over $\vec{p}$ vanishes and we therefore obtain

$$
P\left(\nu_{\alpha} \rightarrow \nu_{\beta}\right)=\sum_{i}\left|U_{\beta i}\right|^{2}\left|U_{\alpha i}\right|^{2}
$$

Notice that this is the probability for a single neutrino rather than the average probability for several neutrinos states.

As discussed in [13], even if the condition (8) is fulfilled, for a hypothetical detector with energy resolution, $\Delta E_{D}$, smaller than $\sigma_{p}$, the interference 
terms can become relevant provided that $\left(\Delta m^{2} L / p\right)\left(\Delta E_{D} / p\right) \lesssim 1$. Let us discuss how the difference between the two cases can be understood within the formalism discussed in this appendix. To calculate the total probability of detecting $\nu_{\beta}$ at the detector, we first calculated the probability of appearance of $\nu_{\beta}$ at a certain point $(\vec{x})$ of the space and then integrated over $\vec{x}$. The rationale behind this treatment was that in a realistic neutrino detector, the neutrinos are detected through the electroweak interactions whose range (i.e., $m_{W}^{-1} \sim 10^{-16} \mathrm{~cm}$ ) is much smaller than the typical size of the wavepacket. In terms of quantum mechanics, to a good approximation, the eigenstates of a realistic detection operator are $\left|\nu_{\beta} ; x, t\right\rangle$. In contrast, the eigenstates of a hypothetical detector with an energy resolution of $\Delta E_{D}$ are states described by $\left|\nu_{\beta}, D\right\rangle \equiv \int g_{D}(\vec{p})\left|\nu_{\beta} ; \vec{p}\right\rangle d^{3} p\left(\neq\left|\nu_{\beta} ; \vec{x}, L\right\rangle\right)$, where $g_{D}(\vec{p})$ is a function of momentum with width $\Delta E_{D}$. Thus, with such a detector, the total probability is given by an integral over $\left|\left\langle\nu_{\beta} ; D \mid \nu_{\alpha}\right\rangle\right|^{2}$ rather than by Eq. (58).

\section{Acknowledgement}

We are grateful to Joern Kersten, Georg Raffelt and Z. Z. Xing for useful discussions. Y.F. would like to thank ICTP where part of this work was done.

\section{References}

[1] A. Achterberg et al. [IceCube Collaboration], Phys. Rev. D 75 (2007) 102001 [arXiv:astro-ph/0611063].

[2] http://icecube.wisc.edu/

[3] B. Zhang and P. Meszaros, Int. J. Mod. Phys. A 19 (2004) 2385 [arXiv:astro-ph/0311321]; T. Piran, Rev. Mod. Phys. 76 (2004) 1143 [arXiv:astro-ph/0405503].

[4] P. Meszaros and E. Waxman, Phys. Rev. Lett. 87 (2001) 171102 [arXiv:astro-ph/0103275]. 
[5] S. Razzaque, P. Meszaros and E. Waxman, Phys. Rev. Lett. 93 (2004) 181101 [Erratum-ibid. 94 (2005) 109903] [arXiv:astro-ph/0407064].

[6] S. Ando and J. F. Beacom, Phys. Rev. Lett. 95 (2005) 061103 [arXiv:astro-ph/0502521].

[7] F. Halzen and A. O'Murchadha, arXiv:0802.0887 [astro-ph]; F. W. Stecker, Phys. Rev. D 72 (2005) 107301 [arXiv:astro-ph/0510537]; F. W. Stecker, C. Done, M. H. Salamon and P. Sommers, Phys. Rev. Lett. 66 (1991) 2697 [Erratum-ibid. 69 (1992) 2738].

[8] V. S. Berezinsky and G. T. Zatsepin, Yad. Fiz. 11 (1970) 200.

[9] H. Athar, M. Jezabek and O. Yasuda, Phys. Rev. D 62 (2000) 103007 [arXiv:hep-ph/0005104]; S. Pakvasa, AIP Conf. Proc. 542 (2000) 99 [arXiv:hep-ph/0004077].

[10] Y. Farzan and A. Y. Smirnov, Phys. Rev. D 65 (2002) 113001 [arXiv:hep-ph/0201105].

[11] A. Bandyopadhyay et al. [ISS Physics Working Group], arXiv:0710.4947 [hep-ph].

[12] J. F. Beacom, N. F. Bell, D. Hooper, S. Pakvasa and T. J. Weiler, Phys. Rev. D 69 (2004) 017303 [arXiv:hep-ph/0309267]; P. D. Serpico and M. Kachelriess, Phys. Rev. Lett. 94 (2005) 211102 [arXiv:hepph/0502088]; P. D. Serpico, Phys. Rev. D 73 (2006) 047301 [arXiv:hepph/0511313]; W. Winter, Phys. Rev. D 74 (2006) 033015 [arXiv:hep$\mathrm{ph} / 0604191]$.

[13] K. Kiers, S. Nussinov and N. Weiss, Phys. Rev. D 53 (1996) 537 [arXiv:hep-ph/9506271].

[14] L. Stodolsky, Phys. Rev. D 58 (1998) 036006 [arXiv:hep-ph/9802387].

[15] E. Waxman and J. N. Bahcall, Phys. Rev. Lett. 78 (1997) 2292 [arXiv:astro-ph/9701231]; E. Waxman and J. N. Bahcall, Phys. Rev. D 59 (1999) 023002 [arXiv:hep-ph/9807282]. 
[16] M. S. Turner and L. M. Widrow, Phys. Rev. D 37 (1988) 2743; M. A. Ganjali, JHEP 0509 (2005) 004 [arXiv:hep-th/0509032]; B. A. Bassett, G. Pollifrone, S. Tsujikawa and F. Viniegra, Phys. Rev. D 63 (2001) 103515 [arXiv:astro-ph/0010628]; A. C. Davis, M. Lilley and O. Tornkvist, Phys. Rev. D 60 (1999) 021301 [arXiv:astro-ph/9904022]; A. Omar, K. R. Anantharamaiah, M. Rupen and J. Rigby, arXiv:astro$\mathrm{ph} / 0111268$.

[17] M. Maltoni and T. Schwetz, Phys. Rev. D 76 (2007) 093005 [arXiv:0705.0107 [hep-ph]].

[18] J. G. Learned and K. Mannheim, Ann. Rev. Nucl. Part. Sci. 50 (2000) 679; G. Ingelman and M. Thunman, Phys. Rev. D 54 (1996) 4385 [arXiv:hep-ph/9604288]; C. Hettlage, K. Mannheim and J. G. Learned, Astropart. Phys. 13 (2000) 45 [arXiv:astro-ph/9910208].

[19] W.-M. Yao, et al., Journal of Physics G 33 (2006) 1.

[20] S. W. Hawking, Commun. Math. Phys. 43 (1975) 199; Phys. Rev. D 14 (1976) 2460.

[21] S. B. Giddings and A. Strominger, Nucl. Phys. B 307 (1988) 854.

[22] G. L. Fogli, E. Lisi, A. Marrone, D. Montanino and A. Palazzo, arXiv:0704.2568 [hep-ph].

[23] E. Lisi, A. Marrone and D. Montanino, Phys. Rev. Lett. 85 (2000) 1166 [arXiv:hep-ph/0002053].

[24] D. Hooper, D. Morgan and E. Winstanley, Phys. Lett. B 609 (2005) 206 [arXiv:hep-ph/0410094].

[25] T. Ohlsson, Phys. Lett. B 502 (2001) 159 [arXiv:hep-ph/0012272].

[26] H. B. J. Koers and R. A. M. Wijers, arXiv:0711.4791 [astro-ph]. 